\title{
Política pública sobre capacitación y empleo en Chile: Inclusión/exclusión de una fuerza laboral que envejece
}

\author{
Nora Gray \\ Pontificia Universidad Católica de Valparaíso, Viña del Mar, Chile. \\ Email: ngrayg@gmail.com \\ Cynthia Basualto \\ Pontificia Universidad Católica de Chile, Santiago, Chile. \\ Email: cdbasualto@uc.cl

\section{Vicente Sisto} \\ Pontificia Universidad Católica de Valparaíso, Viña del Mar, Chile. \\ Email: vicente.sisto@ucv.cl
}

\begin{abstract}
Resumen: Nuestra sociedad está envejeciendo, muchas personas se verán obligados a trabajar hasta avanzada edad. Desde el punto de vista laboral, se requieren de políticas públicas inclusivas de la fuerza laboral a medida que envejece ofreciendo oportunidades de capacitación y fomento del empleo. Este estudio exploratorio analizó la oferta de los programas vigentes del Servicio Nacional de Capacitación y Empleo respecto a si promueven o no la inclusión de trabajadores mayores. Se realizó una caracterización de los programas considerando antigüedad, focalización, presupuesto, población objetivo, rango etario, periodo de gobierno. Se clasificaron de acuerdo a su grado de inclusión en: no inclusivos, parcialmente inclusivos e inclusivos. De los trece programas analizados solamente cinco son realmente inclusivos. Se propone revisar los demás programas de modo de buscar ajustes o cambios que pudieran ampliar su grado de inclusión, en pos de mayor equidad para las personas de toda edad en nuestro país.
\end{abstract}

Palabras clave: Trabajo, formación, ciclo de vida, análisis documental.

\section{Public policy on training and employment in Chile: Inclusion / exclusion of an aging workforce}

\footnotetext{
Abstract: Our society is aging, many people will be forced to work until old age. From a labor standpoint, inclusive public policies of the workforce are required as it grows old by offering training and employment promotion opportunities. This exploratory study analyzed the offer of the current programs of the National Service of Training and Employment as to whether or not they promote the inclusion of older workers. A characterization of the programs was carried out considering seniority, targeting, budget, target population, age range,
} 
government period. They were classified according to their degree of inclusion in: non-inclusive, partially inclusive and inclusive. Among the thirteen programs analyzed, only five are truly inclusive. It is proposed to review the other programs in order to seek adjustments or changes that could increase their degree of inclusion, in order to reach better equity for people of all ages in our country.

Keywords: Work, training, life cycle, documentary analysis

\section{Política pública de formação e emprego no Chile: Inclusão/ exclusão da força de trabalho que está em processo de envelhecimento}

Resumo: Nossa sociedade está envelhecendo, muitas pessoas serão forçadas a trabalhar até a velhice. Do ponto de vista do trabalho, são necessárias políticas públicas inclusivas da força de trabalho à medida que envelhecem, oferecendo oportunidades de treinamento e promoção de emprego. Este estudo exploratório analisou a oferta dos atuais programas do Serviço Nacional de Formação e Emprego sobre a promoção ou não da inclusão de trabalhadores mais velhos. Foi realizada uma caracterização dos programas considerando a antiguidade, segmentação, orçamento, população-alvo, faixa etária, período do governo. Eles foram classificados de acordo com seu grau de inclusão em: não inclusivo, parcialmente inclusivo e inclusivo. Dos treze programas analisados, apenas cinco são verdadeiramente inclusivos. Propõese rever os outros programas para buscar ajustes ou mudanças que possam aumentar seu grau de inclusão, a fim de aumentar a equidade para pessoas de todas as idades em nosso país. mental

Palavras-chave: Trabalho, treinamento, ciclo de vida, análise docu-

\section{Introducción}

En una sociedad que envejece como la nuestra, a medida que se transforma la composición de la población y la proporción de adultos mayores aumenta, con alta probabilidad enfrentaremos un escenario futuro con muchas personas que estarán activas a edad muy avanzada. Tal como muestran los datos actuales sobre América Latina y el Caribe, los sistemas de salud y de previsión enfrentan el desafío de responder las necesidades de esta generación que se encuentra cercana a la edad de jubilación y que ha ahorrado poco en términos previsionales: en la región, solo 45 de cada 100 trabajadores están contribuyendo o están afiliados a un plan de pensiones (OIT, 2016; CEPAL, 2012).

Uno de los grandes debates en el presente es la función de la seguridad social en una sociedad que envejece. Esta situación es mucho más difícil para los países en desarrollo, donde los niveles de cobertura y prestaciones siguen siendo insuficientes (OCDE, 2015). El envejecimiento activo ha surgido como una herramienta política importante para abordar la sostenibilidad de la seguridad social en algunos países, especialmente en 
Europa, lo cual debiera considerar la reactivación de los adultos mayores para los mercados de trabajo. El concepto de envejecimiento activo fue desarrollado originalmente por la Organización Mundial de la Salud el año 2002, se define como: “... el proceso de optimización de oportunidades para la salud, la participación y la seguridad con el fin de mejorar la calidad de vida a medida que la gente envejece.” (WHO, 2002). La OMS enfatiza entonces la relación estrecha que existe entre la actividad y la salud, subrayando la importancia de mejorar la calidad de vida en personas mayores mediante el mantenimiento del bienestar mental y físico a través de todo el ciclo vital.

En Chile a partir de los años 90 se ha observado la implementación de diversas políticas que apuntan a relevar la necesidad de enfrentar el fenómeno del envejecimiento poblacional en nuestro país. En términos generales, podría señalarse que el principal responsable de este tema en el ámbito público, el Servicio Nacional del Adulto Mayor (SENAMA), ha promovido una política que apunta a incentivar el envejecimiento positivo dentro de la sociedad chilena. En específico, resalta la importancia de proteger la salud funcional de las personas mayores, mejorar su integración y participación social, así como de incrementar su bienestar subjetivo (Chile, 2012). El cuadro 1 resume algunos de los principales hitos en el desarrollo de políticas públicas con respecto a las personas mayores en el país.

\section{Cuadro1 \\ Hitos en el desarrollo de políticas públicas para el envejecimiento de la población.}

\begin{tabular}{|c|l|}
\hline 1995 & $\begin{array}{l}\text { Siendo Presidente de la República Eduardo Frei Ruiz- } \\
\text { Tagle, fue creada la Comisión Nacional para el Adulto } \\
\text { Mayor, órgano asesor del Presidente en la formula- } \\
\text { ción de las políticas, planes y programas para el adul- } \\
\text { to mayor. }\end{array}$ \\
\hline 2002 & $\begin{array}{l}\text { Congreso Nacional aprueba el Proyecto de Ley de } \\
\text { Creación del Servicio Nacional del Adulto Mayor, cuya } \\
\text { ley No 19.828 es promulgada el 17 de septiembre de } \\
\text { 2002. En enero de 2003, SENAMA inicia sus funcio- } \\
\text { nes. }\end{array}$ \\
\hline $2002-2012$ & $\begin{array}{l}\text { El Estado ha diseñado e implementado programas y } \\
\text { beneficios dirigidos a responder a las necesidades del } \\
\text { adulto mayor, en los diversos ámbitos que permitan } \\
\text { mejorar su calidad de vida, su bienestar económico, } \\
\text { social y cultural. }\end{array}$ \\
\hline
\end{tabular}




\begin{tabular}{|l|l|}
\hline 2012 & $\begin{array}{l}\text { Creación “Política Integral de Envejecimiento Positi- } \\
\text { vo para Chile 2012-2025”. } \\
\text { Las áreas que abarca esta política son: turismo, salud, } \\
\text { trabajo, educación, ingresos familiares, vivienda, cul- } \\
\text { tura, transporte, participación social y justicia. }\end{array}$ \\
\hline 2013 & $\begin{array}{l}\text { Diferentes Ministerios y Servicios Públicos comenza- } \\
\text { ron a participar en el desarrollo en conjunto de pro- } \\
\text { gramas y beneficios dedicados al adulto mayor. }\end{array}$ \\
\hline
\end{tabular}

Fuente: SENCE (2017) y Ganga, Piñones, González y Rebagliati (2016)

Chile enfrenta una compleja situación en materia de pensiones, caracterizada por una baja cobertura, una baja densidad de cotizaciones y una tasa de reemplazo que con sólo un 33,1\% en las mujeres y un 37,7\% en los hombres está entre las más bajas de los países de la OCDE (OECD, 2016; Mesa-Lago, 2015; Sojo, 2014). Se configura así un panorama muy desafiante en materia laboral. Sin duda, los trabajadores chilenos de hoy necesitarán seguir activos hasta una edad mucho más avanzada ya que, con alta probabilidad, en los próximos años la edad legal de jubilación se elevará, como ha sido la tónica ya en otras latitudes. Estudios recientes han mostrado que ya es un hecho que muchos chilenos siguen trabajando más allá de la edad legal de jubilación y que nuestro país presenta una edad efectiva de retiro promedio elevada tanto para hombres (70,9 años) como para mujeres (70,3 años), una de las más altas entre los países que conforman la OECD (OECD, 2015). La Encuesta de Calidad de Vida en la Tercera Edad, que se realiza en conjunto entre la Pontificia Universidad Católica de Chile, el Servicio Nacional del Adulto Mayor y Caja Los Andes en su última versión señala que la motivación de la mayoría de los trabajadores mayores de 60 años para seguir trabajando (66\%) es la necesidad económica (Programa Adulto Mayor UC y Centro UC Estudios de Vejez y Envejecimiento, 2017).

Datos recientes muestran de qué modo el segmento de adultos mayores ha ido ganando terreno entre las personas en edad de trabajar en los últimos años en nuestro país. Entre el año 2010 y el 2015 (trimestre octubrediciembre) los adultos mayores aumentaron desde el 20,2\% de la población en edad de trabajar al 23,3\% (Bravo, 2016). Estudios internacionales documentan que este segmento de la población tiende a insertarse en el mercado laboral proporcionalmente más en empleos por cuenta propia y menos en empleos asalariados (Eurofound, 2012), lo cual también se observa en Chile (Bravo, 2016; Marcel \& Naudon, 2016; Vives, Molina, Gray, \& González, 2017).

Una idea que cobra fuerza a la hora de integrar activamente a las personas en el mundo del trabajo es la de inclusión. El concepto de inclusión laboral tiene su contraparte en el de exclusión laboral. En palabras de Weller (2001), esto alude a "procesos que permiten el acceso de una parte 
de la población a empleos productivos con condiciones laborales favorables o adecuadas, mientras que otra parte de la población no tiene acceso a este tipo de empleo” (p. 13). Autores como Caldera (2014) señalan que una mayor inclusión laboral puede contribuir a disminuir los niveles de pobreza, promoviendo así que los grupos más vulnerables puedan acceder a mejores niveles de salarios.

En materia de inclusión vinculada al envejecimiento, en Chile se ha desarrollado hasta la fecha cuatro versiones de la Encuesta Nacional de Inclusión y Exclusión de las Personas Mayores que se realiza en forma conjunta entre SENAMA y la Universidad de Chile ${ }^{1}$. Los resultados más recientes han planteado que es fundamental enfrentar los prejuicios que existen en la población sobre el proceso de envejecimiento para poder incorporarlos a la actividad social regular; los chilenos tienen una evaluación crítica respecto de la preparación institucional ante el envejecimiento poblacional y no asumen su propia preparación para enfrentar su vejez (Thumala, Arnold, Massad, \& Herrera, 2015). Diversos autores plantean que la discriminación y la exclusión en el campo laboral se puede evitar si los adultos mayores están suficientemente capacitados y actualizados en conocimientos (Eichhorst, Boeri, Braga, Coen, Galasso, Gerard, \& Steiber, 2013; Cepeda, 2010; Ghosheh, 2008).

\section{La capacitación como herramienta para la permanencia en el mercado de trabajo}

Weller (2014) señala que las las políticas activas de mercado de trabajo o políticas activas de generación de empleo se pueden sintetizar en: 1) las políticas para generar empleo, en especial en periodos de crisis económica y/o medioambiental y 2) las políticas destinadas a mejorar la calidad de los empleos existentes, en especial los de baja productividad, y aumentar la empleabilidad de los trabajadores mediante herramientas como la capacitación laboral, la formación profesional en sus distintas modalidades y la acreditación o certificación de competencias laborales. Una de las herramientas que se han tornado imprescindibles para promover la empleabilidad en este contexto es la capacitación (Didier, Pérez, \& Valdenegro, 2013).

La capacitación sirve no solamente para incrementar el conocimiento personal aplicado al trabajo, sino que también para re-orientar el curso de una vida laboral, mantenerse vigente y reinventarse. Los diferentes modelos de capacitación tienen distintas poblaciones objetivo, dependiendo del nivel de preparación de los individuos para el lugar de trabajo en cuanto a habilidades (blandas o socioemocionales, competencias matemáticas y lingu?ísticas, técnicas), a la experiencia, la edad y al objetivo de la capacitación de por sí (Didier \& Cox, 2012). Cuando se plantea en otras latitudes el trabajo de los adultos mayores, uno de los elementos sustantivos para facilitar la inserción laboral es la capacitación, la protección y fomento del empleo. Didier et al. (2013) que utilizaron datos de la Encuesta de Caracteri- 
zación Socioeconómica (Casen) para describir el funcionamiento del sistema de capacitación en Chile en las últimas dos décadas, observaron que para el 2009 la participación total en instancias de educación para el trabajo entre personas mayores se aproxima a un $22 \%$.

\section{Fomento del empleo y la capacitación en Chile desde el ámbito público: el Servicio Nacional de Capacitación y el Empleo (SENCE)}

El SENCE se creó el año 1976 constituyéndose como un organismo técnico descentralizado del Estado que se relaciona con el gobierno a través del Ministerio del Trabajo y Previsión Social. La misión del SENCE es contribuir al incremento de la productividad en la economía a través de medidas que impulsen la capacitación de los trabajadores en las empresas, y también de las personas de bajos ingresos que no participan en el mercado laboral (SENCE, s. f).

El SENCE cuenta en la actualidad con diversos programas para el fomento del empleo y la capacitación de diversos grupos de la población en el país. Sus principales objetivos son aumentar la competitividad de las empresas y la empleabilidad de las personas, por medio de la aplicación de políticas públicas y programas dirigidos al mercado de la capacitación, intermediación laboral, emprendimientos y subsidios ligados a un proceso de formación permanente. De acuerdo a datos obtenidos del SENCE en el año 2011, el estado invierte alrededor de un $82 \%$ de los recursos destinados a capacitación, en tanto los privados aportan una cifra cercana al 18\% (Didier \& Cox, 2012). Según la información reseñada, la mayor parte de las acciones de capacitación realizadas tienen como beneficiarios a trabajadores calificados, administrativos, y profesionales. Un estudio publicado recientemente señala que son las grandes empresas las que tienden a realizar más capacitaciones con un 96,8\%; les siguen las medianas empresas con un 90,1\%, las pequeñas empresas con un $72,4 \%$ y las microempresas con un $45,8 \%$ (Mardones \& Sepúlveda, 2017).

El año 2011 se creó una Comisión Revisora del Sistema de Capacitación e Intermediación Laboral que evaluó la situación en el área en el país para proponer ajustes y cambios. La Comisión puso de manifiesto la carencia de información confiable que permitiera evaluar el logro de los programas dirigidos a las poblaciones más vulnerables, lo que fue claramente identificado como uno de los grandes déficits de programas dirigidos a jóvenes y mujeres. En palabras textuales, se señala en el documento de Informe Final que emitió la Comisión que tal déficit significa "conducir la política pública a ciegas, sin poder conocer si lo que se hace tiene algún impacto en la población objetivo" (Comisión Revisora del Sistema de Capacitación e Intermediación Laboral, 2011, p. 7).

En un interesante análisis comparativo reciente sobre el funciona- 
miento de un tipo específico de programa de capacitación -Aprendices ${ }^{2}$ en diversos países, Fazio, Fernández-Coto, y Ripani (2016) muestran que algunos programas o sistemas como los de Australia, Alemania, Canadá, Costa Rica, Inglaterra, India, Malta, Colombia y Estados Unidos admiten personas de todas las edades, mientras que en Brasil, Chile y Perú existen límites de 24, 25 y 29 años, respectivamente. En esos casos, por lo tanto, la prioridad de esos programas estaría en resolver los desafíos de la empleabilidad juvenil que afrontan los países, lo cual difiere de lo que en Europa se ha venido planteando y concretando respecto a políticas pública sobre la materia en los últimos años.

Las naciones del mundo adoptaron en el año 2015 la Agenda 2030 de Desarrollo Sostenible, cuyo octavo objetivo plantea "promover el crecimiento económico sostenido, inclusivo y sostenible, el empleo pleno y productivo y el trabajo decente para todos” (CEPAL, 2017, p.29). El compromiso que los países han adquirido al suscribir esta agenda tiene implicancias respecto a temas laborales con relación a inclusión, sostenibilidad y trabajo decente a lo largo de todo el ciclo vital, por tanto se puede concluir que esto incluye también a los trabajadores de mayor edad.

El objetivo de este artículo es analizar si la institucionalidad pública en Chile en materia de capacitación y fomento del empleo de los trabajadores, representada en el SENCE, promueve o no la inclusión de la fuerza laboral que envejece, a partir de una revisión de los programas que SENCE ofrece en la actualidad.

\section{Metodología}

\section{Tipo de estudio y características de la muestra}

Este es un estudio cualitativo, de análisis documental (Atkinson \& Coffey, 2006), desde el cual se entienden los distintos informes de la política como actores que legitiman autoridad y validan formas de poder, permitiendo estandarizar y categorizar a la población que envejece en relación con el empleo.

Esta investigación se apoya e inspira metodológicamente en el trabajo, entre otros, de Sisto y Fardella (2011), Pavez, Aguilar, Peña, Bilbao, Oriol, Ortúzar y Torres (2016) y Rivera (2016), que han estudiado políticas públicas a partir del análisis documental. Tal como señala Rivera (2016):

"La relevancia del análisis radica en que los textos, sean documentos oficiales o discursos de autoridades, sirven para presentar una lógica de gestión, actuar en el terreno local, interpelar a los actores, moldear relaciones, prescribir acciones, permitiendo de esta manera estandarizar y categorizar a los sujetos” (Rivera, 2016, p. 198). 
Polis, Revista Latinoamericana, $N^{\circ}$ 48, 2017

\section{Muestra}

Los documentos que se analizaron fueron programas del ámbito de la capacitación y el empleo gestionados por el Servicio Nacional de Capacitación y Empleo (SENCE, 2017). Se tomó la decisión de focalizar el estudio en esta institución puesto que es la principal entidad pública encargada de mejorar la empleabilidad de trabajadores ocupados/desocupados y vulnerables a lo largo de su vida laboral, por medio de una gestión articulada entre la orientación, capacitación e intermediación laboral (SENCE, s. f). La función que cumple esta institución en el contexto laboral la ubica en una posición clave si se busca analizar la dimensión de inclusión/exclusión de la fuerza laboral respecto a la capacitación y el empleo, a toda edad.

Los programas analizados cumplieron con los siguientes criterios de inclusión y exclusión:

- Criterios de inclusión: Todo programa vigente al año 2017 y disponible en la sección de descripción de programas SENCE de la página web oficial.

- Criterios de exclusión: Todo programa que se encuentra descontinuado o no vigente al año 2017 y que no esté disponible en la sección de descripción de programas SENCE de la página web oficial.

SENCE clasifica los programas en 4 categorías que se sistematizan en el siguiente cuadro:

\section{Cuadro 2 \\ Clasificación de los programas especiales de apoyo de SENCE}

\begin{tabular}{|l|l|}
\hline \multicolumn{1}{|c|}{$\begin{array}{c}\text { Categorias de Programas } \\
\text { (Linea de intervención) }\end{array}$} & \multicolumn{1}{c|}{$\begin{array}{c}\text { Programas especiales de Apoyo } \\
\text { (vigente año 2017) }\end{array}$} \\
\hline Apoyo al emprendimiento & Bono empresa y negocio \\
\hline & +Capaz \\
& Impulsa personas \\
& Aprendices \\
& Capacitación en oficios \\
Evaluación y certificación de competencias \\
Capacitación & laborales \\
& Capacitación en oficios sectoriales \\
& Becas laborales \\
& Transferencias al sector público \\
& Becas fondo cesantía solidario \\
\hline Empleo & Fortalecimiento OMIL \\
\hline Subsidios & Bono al trabajo de la mujery subsidio al empleo \\
& joven \\
\hline
\end{tabular}

Fuente: SENCE (2017) 


\section{Instrumentos o técnicas de producción de información}

La técnica de producción de información fue el análisis de documentos oficiales que corresponden a programas que operacionalizan políticas públicas en materias de capacitación y empleo. Desde el punto de vista metodológico, se sistematizó la información de los programas con base en categorías que emergieron de la estructura con que se describen. Estas son:

- Antigüedad.

- Focalización.

- Presupuesto.

- Población objetivo.

- Rango/límite etario.

- Periodo de gobierno.

La elección de dichas categorías para el análisis de los programas se justifica por el aporte potencial que representan para el eje de este estudio, que es la inclusión/exclusión de la dimensión de envejecimiento de la población laboral. En el cuadro 3 se observa la descripción de cada categoría.

\section{Cuadro 3 \\ Categorías de análisis y su descripción}

\begin{tabular}{|c|c|}
\hline Categorías de Análisis & Descripción \\
\hline Antigüledad & $\begin{array}{l}\text { Año de creación y tiempo en que lleva vigente el } \\
\text { programa. }\end{array}$ \\
\hline Focalización & $\begin{array}{l}\text { Beneficiarios que establece el programa (grupo } \\
\text { social específico que cumpla con ciertas } \\
\text { características de vulnerabilidad, sexo y/o edad). }{ }^{3}\end{array}$ \\
\hline Presupuesto & $\begin{array}{l}\text { Monto de dinero asignado en un año específico } \\
\text { según la Ley de presupuesto vigente. Según datos del } \\
\text { año } 2016 \text {. }\end{array}$ \\
\hline Población objetivo & $\begin{array}{l}\text { Cantidad de personas al cual los programas buscan } \\
\text { beneficiar. Según datos del año } 2016 \text {. }\end{array}$ \\
\hline Rango/límite etario & $\begin{array}{l}\text { Edad mínima y máxima establecida en el programa } \\
\text { para el acceso. }\end{array}$ \\
\hline Periodo de gobierno & $\begin{array}{l}\text { Presidente y gobierno de turno correspondiente al año } \\
\text { de creación del programa. }\end{array}$ \\
\hline Inclusión/exclusión & $\begin{array}{l}\text { Grado en que el programa está abierto a personas que } \\
\text { trabajan en la medida que envejecen: inclusivo (para } \\
\text { todo rango etario), parcialmente inclusivo (con acceso } \\
\text { hasta cierta edad o bajo ciertas condiciones), no } \\
\text { inclusivo (programa limitado a un rango o grupo } \\
\text { específico solamente) }\end{array}$ \\
\hline
\end{tabular}

Fuente: Elaboración propia con base en información SENCE (2017) 


\section{Resultados}

\section{Objetivos y focalización de los programas}

SENCE al año 2017 gestiona 13 programas que tienen como objetivo intervenir en diferentes temáticas, así como también beneficiar a diversos públicos objetivo. Tal como se muestra en el cuadro 3, cada programa posee un objetivo general y criterios de focalización.

\section{Cuadro 4}

\section{Programas SENCE revisados, sus objetivos y criterios de focalización}

\begin{tabular}{|c|c|c|}
\hline Nombre programa & Objetivo & $\begin{array}{c}\text { Focalización } \\
\text { de los programas }\end{array}$ \\
\hline $\begin{array}{l}\text { Bono Empresa y Ne- } \\
\text { gocio }\end{array}$ & $\begin{array}{l}\text { Mejorar productividad } \\
\text { de micro-pequeños } \\
\text { empresarios con capa- } \\
\text { citación y beca-bono de } \\
\text { capacitación. }\end{array}$ & $\begin{array}{l}\text { Dueños, socios y repre- } \\
\text { sentantes legales de } \\
\text { micro y pequeñas em- } \\
\text { presas } \\
\text { Trabajadores por cuen- } \\
\text { ta propia } \\
\text { Personas pertenecien- } \\
\text { tes a registros especia- } \\
\text { les } \\
\text { No explicita rango etario } \\
\text { ni grupo social especí- } \\
\text { fico. }\end{array}$ \\
\hline + Capaz & $\begin{array}{l}\text { Aumentar tasa de par- } \\
\text { ticipación laboral en } \\
\text { mujeres, jóvenes y } \\
\text { personas con disca- } \\
\text { pacidad }\end{array}$ & $\begin{array}{l}\text { Hombres entre } 18 \text { y } 29 \\
\text { años de edad y mujeres } \\
\text { de entre } 18 \text { y } 64 \text { años } \\
\text { de edad. } \\
\text { Personas en situación de } \\
\text { discapacidad entre } 18 \text { y } \\
50 \text { años. } \\
\text { Personas infractoras de } \\
\text { ley entre } 14 \text { y } 64 \text { años }\end{array}$ \\
\hline Aprendices & $\begin{array}{l}\text { Generar empleabilidad } \\
\text { y productividad a tra- } \\
\text { vés del desarrollo de } \\
\text { competencias relacio- } \\
\text { nadas a una ocupación. }\end{array}$ & $\begin{array}{l}\text { Jóvenes desocupados } \\
\text { mayores de } 15 \text { y meno- } \\
\text { res de } 25 \text { años. } \\
\text { Para población en situa- } \\
\text { ción de discapacidad no } \\
\text { hay límite de edad. }\end{array}$ \\
\hline Impulsa Persona & $\begin{array}{l}\text { Mejorar capital huma- } \\
\text { no, a través de capacita- } \\
\text { ción, evaluación y certifi- } \\
\text { cación de competencias, } \\
\text { ofreciendo descuentos en } \\
\text { pago de impuestos. }\end{array}$ & $\begin{array}{l}\text { Personas mayores de } \\
15 \text { años. } \\
\text { Pertenecer a la fuerza de } \\
\text { trabajo. } \\
\text { No explicita rango etario } \\
\text { ni grupo social específico }\end{array}$ \\
\hline
\end{tabular}




\begin{tabular}{|c|c|c|}
\hline $\begin{array}{l}\text { Capacitación en Ofi- } \\
\text { cios registro especial }\end{array}$ & $\begin{array}{l}\text { Generar competencias } \\
\text { en personas vulnera- } \\
\text { bles, para aumentar su } \\
\text { empleabilidad, y en } \\
\text { trabajadores indepen- } \\
\text { dientes aumentar sus } \\
\text { ingresos. }\end{array}$ & $\begin{array}{l}\text { Hombres y mujeres de } \\
\text { entre } 16 \text { a } 65 \text { años que } \\
\text { pertenezcan al } 60 \% \text { más } \\
\text { vulnerable de la pobla- } \\
\text { ción }\end{array}$ \\
\hline $\begin{array}{l}\text { Evaluación y Certifica- } \\
\text { ción de Competencias } \\
\text { Laborales }\end{array}$ & $\begin{array}{l}\text { Reconocer competen- } \\
\text { cias de las personas, in- } \\
\text { dependientemente de } \\
\text { cómo haya sido adqui- } \\
\text { rido. }\end{array}$ & $\begin{array}{l}\text { Hombres y mujeres } \\
\text { mayores de } 18 \text { años. } \\
\text { No explicita límite } \\
\text { etario específico. }\end{array}$ \\
\hline $\begin{array}{l}\text { Capacitación en oficios } \\
\text { sectoriales }\end{array}$ & $\begin{array}{l}\text { Generar competencias } \\
\text { laborales de acuerdo a } \\
\text { los requerimientos de } \\
\text { los sectores producti- } \\
\text { vos con el propósito de } \\
\text { aumentar la probabili- } \\
\text { dad de encontrar un } \\
\text { empleo. }\end{array}$ & $\begin{array}{l}\text { Tener entre } 16 \text { y } 65 \\
\text { años. } \\
\text { Tener la edad exigida por } \\
\text { el sector productivo } \\
\text { Pertenecer al } 60 \% \text { más } \\
\text { vulnerable de la pobla- } \\
\text { ción }\end{array}$ \\
\hline Becas Laborales & $\begin{array}{l}\text { Mejorar competencias } \\
\text { laborales y facilitar ac- } \\
\text { ceso a empleo o activi- } \\
\text { dad productiva en mu- } \\
\text { jeres y jóvenes }\end{array}$ & $\begin{array}{l}\text { Hombres y mujeres } \\
\text { mayores de } 17 \text { años. } \\
\text { Pertenecer al } 80 \% \text { más } \\
\text { vulnerable de la pobla- } \\
\text { ción. } \\
\text { No especifica edad de } \\
\text { corte. } \\
\text { En línea de inclusión la- } \\
\text { boral explicita: benefi- } \\
\text { ciar a personas mayo- } \\
\text { res de } 64 \text { años. }\end{array}$ \\
\hline $\begin{array}{l}\text { Transferencias al Sec- } \\
\text { tor Público }\end{array}$ & $\begin{array}{l}\text { Facilitar la inserción } \\
\text { laboral de las personas } \\
\text { dependientes de las } \\
\text { instituciones en conve- } \\
\text { nio con SENCE, gene- } \\
\text { rando nuevas compe- } \\
\text { tencias. }\end{array}$ & $\begin{array}{l}\text { Fuerzas Armadas de } \\
\text { Chile: Conscriptos. } \\
\text { Gendarmería de Chile: } \\
\text { Personas imputadas, } \\
\text { procesadas y condena- } \\
\text { das de los sistemas pe- } \\
\text { nitenciarios y jóvenes. } \\
\text { Servicio Nacional de } \\
\text { Menores: Adolescentes } \\
\text { de } 14 \text { años } \\
\text { Instituto de Desarrollo } \\
\text { Agropecuario: Peque- } \\
\text { ños productores agríco- } \\
\text { las. } \\
\text { No explicita rango etario } \\
\text { específico }\end{array}$ \\
\hline
\end{tabular}




\begin{tabular}{|c|c|c|}
\hline $\begin{array}{l}\text { Becas Fondo Cesantía } \\
\text { Solidaria }\end{array}$ & $\begin{array}{l}\text { Aumentar empleabi- } \\
\text { lidad, movilidad, } \\
\text { reinserción de perso- } \\
\text { nas cesantes mediante } \\
\text { capacitación }\end{array}$ & $\begin{array}{l}\text { Cesantes beneficiarios } \\
\text { del Fondo de Cesantía } \\
\text { Solidario. } \\
\text { No explicita rango etario } \\
\text { ni grupo social especí- } \\
\text { fico }\end{array}$ \\
\hline $\begin{array}{l}\text { F o rta le c i m i e n to } \\
\text { OMIL (Oficinas Mu- } \\
\text { nicipales de Informa- } \\
\text { ción Laboral) }\end{array}$ & $\begin{array}{l}\text { Desarrollar el sistema } \\
\text { de intermediación, en- } \\
\text { tregando recursos y } \\
\text { metodología de trabajo } \\
\text { a las OMIL, para lo- } \\
\text { grar la inserción labo- } \\
\text { ral en un empleo for- } \\
\text { mal. }\end{array}$ & $\begin{array}{l}\text { Todas las comunas del } \\
\text { país, que tengan Ofici- } \\
\text { na Municipal de Infor- } \\
\text { mación Laboral consti- } \\
\text { tuida. } \\
\text { No explicita rango etario } \\
\text { ni grupo social especí- } \\
\text { fico }\end{array}$ \\
\hline $\begin{array}{l}\text { Bono en el Trabajo de } \\
\text { la Mujer }\end{array}$ & $\begin{array}{l}\text { Mejorar condiciones } \\
\text { laborales en mujer en } \\
\text { situación de vulnerabi- } \\
\text { lidad entre } 25 \text { y } 59 \\
\text { años }\end{array}$ & $\begin{array}{l}\text { Mujeres entre } 25 \text { a } 59 \\
\text { años } \\
\text { Pertenecientes al } 40 \% \\
\text { más vulnerable de la } \\
\text { población }\end{array}$ \\
\hline $\begin{array}{l}\text { Subsidio al Empleo Jo- } \\
\text { ven }\end{array}$ & $\begin{array}{l}\text { Mejorar el ingreso, } \\
\text { condiciones laborales y } \\
\text { la inserción laboral de } \\
\text { los trabajadores entre } \\
18 \text { y } 25 \text { años con bajos } \\
\text { salarios y parte del } \\
40 \% \text { más vulnerable de } \\
\text { la población. }\end{array}$ & $\begin{array}{l}\text { Personas jóvenes entre } \\
18 \text { y menos de } 25 \text { años } \\
\text { Pertenecientes al } 40 \% \\
\text { más vulnerable de la } \\
\text { población }\end{array}$ \\
\hline
\end{tabular}

Fuente: Programas SENCE (2017)

Tal como se observa en el cuadro anterior, más de la mitad de los programas (nueve) se focalizan en capacitación, lo cual reafirma que el lineamiento principal de SENCE es precisamente esa área de intervención. Por otra parte, al observar los programas del resto de las categorías (apoyo al emprendimiento, empleo y subsidios), se aprecia que realizan intervenciones en los ámbitos de incentivos a pequeñas y medianas empresas, entrega de beneficios monetarios y fortalecimiento de la institución pública a cargo de la intermediación laboral.

Se puede señalar que del total de programas analizados siete están focalizados en un grupo social específico y/o con un rango de edad determinado. Estos son:

- Programa + Capaz.

- Programa Aprendices.

- Programa Bono en el Trabajo de la Mujer.

- Programa denominado Capacitación en Oficios registro especial. 
- Subsidio al Empleo Joven.

- Programa Transferencia al Sector Público.

- Capacitación en Oficios sectorial.

El resto de los programas vigentes a la fecha no explicitan como requisito de participación un rango etario en específico. Estos son:

- Becas de Fondo Cesantía Solidaria.

- Bono Empresa y Negocio.

- Becas Laborales ${ }^{4}$.

- Evaluación y Certificación de Competencias Laborales.

- Impulsa Personas.

- Fortalecimiento OMIL ${ }^{5}$.

\section{Presupuesto y población potencial de los programas.}

Los programas analizados entregan información sobre el presupuesto asignado y su población potencial en distintos años. Para poder realizar una comparación entre programas, se ha decidido metodológicamente utilizar el dato presupuestario correspondiente al año 2016, pues algunos programas no poseían datos actualizados para el 2017.

En la siguiente tabla se puede observar el presupuesto asignado y su población potencial para el año 2016, considerando también la antigüedad de cada programa.

\section{Tabla 1 \\ Programas SENCE vigentes, antigüedad presupuesto y población potencial al 2016}

\begin{tabular}{|lrrr|}
\hline \multicolumn{1}{|c}{ Nombre Programa } & $\begin{array}{c}\text { Antigüedad } \\
\text { (años) }\end{array}$ & $\begin{array}{c}\text { Presupuesto 2016 } \\
\text { (miles \$) }\end{array}$ & $\begin{array}{r}\text { Población potencial } \\
2016\end{array}$ \\
\hline Impulsa Persona año 2017 & 41 & Sin información & 8.635 .432 \\
+ Capaz & 3 & 96.380 .996 & 2.032 .709 \\
Bono en el Trabajo de la Mujer año 2017 & 5 & 57.221 .053 & 1.553 .586 \\
Subsidio al Empleo Joven año 2017 & 8 & 55.095 .040 & 773.601 \\
Becas Laborales año 2017 & 18 & 43.116 .600 & 7.935 .447 \\
Capacitación en Oficios registro especial año 2017 & 20 & 18.175 .380 & 6.274 .560 \\
Fortalecimiento OMIL año 2017 & 8 & 5.444 .954 & Sin información \\
Transferencias al Sector Público 2017 & 29 & 3.127 .322 & 265.185 \\
Evaluación y Certificación de Competencias & 9 & 1.686 .509 & 13.578 .306 \\
Laborales año 2017 & 5 & 2.500 .000 & 6.274 .560 \\
Capacitación en Oficios sectorial año & & & 854.169 \\
2017 & 6 & 1.336 .425 & 151.444 \\
Bono Empresa Y Negocio 2017 & 11 & 1.069 .140 & 111.818 \\
Aprendices año 2017 & 10 & 961.950 & \\
Becas Fondo Cesantía Solidaria año 2017 & 5 & \\
\hline
\end{tabular}

Fuente: Elaboración propia a partir de información de SENCE 
En la tabla anterior se observa que el presupuesto asignado no tiene relación directa con la población potencial: los programas con un mayor presupuesto no coinciden con aquellos que tienen una mayor población potencial.

Así también se observa que los tres proyectos a los que se le asigna un monto más elevando en su presupuesto anual, que son $+\mathrm{Ca}-$ paz, Bono en el Trabajo de la Mujer y Subsidio al Empleo Joven, son programas creados y gestionados durante la última década. Más aún, cabe destacar que el programa +Capaz -el más recientemente creado de todos los analizados- es al cual se le ha asignado el mayor presupuesto, con una suma que supera por un amplio margen los $\$ 96.000$ millones de pesos.

La información con que se cuenta para el año 2016 muestra que el programa de Evaluación y Certificación de Competencias Laborales es el que tiene una mayor población objetivo. Esto seguramente se debe a que es uno de los programas más flexibles en sus requisitos de participación, pues sólo declara que las personas deben ser mayores de 18 años, sin hacer diferenciación de género o grupo social en específico. Cabe aclarar que el otro programa que representa un espectro de población amplio es el Impulsa Persona, puesto que está dirigido a personas mayores de 15 años que pertenezcan a la fuerza laboral.

\section{Antigüedad de los programas y gobiernos en que fueron creados}

A partir del año 1976 se comenzó con la creación de programas a cargo de SENCE, que se han ido modificando, eliminando y dando paso a otros nuevos, de los cuales hoy en día sólo trece están vigentes. En efecto, todos estos programas se han iniciado en diferentes gobiernos durante estos 41 años. Del análisis de los programas vigentes y los gobiernos en que se puede inferir un estilo de gestión en capacitación y fomento del empleo durante cada administración.

A continuación, en la Tabla 2 se resumen los programas vigentes a la fecha y los gobiernos responsables detrás de su implementación. 


\section{Tabla 2}

\section{Año y gobiernos en que se iniciaron los programas SENCE}

\begin{tabular}{|c|c|c|}
\hline Nombre Programa & Año de Inicio & Gobiemo \\
\hline $\begin{array}{l}\text { 1. Impulsa Persona año } 2017 \text { (Capacitación) } \\
\text { 2. Transferencias al Sector Público } 2017 \\
\text { (Capacitación) }\end{array}$ & $\begin{array}{l}1976 \\
1988 \\
\end{array}$ & Gobierno Militar \\
\hline $\begin{array}{l}\text { 3. Capacitación en Oficios registro especial } \\
\text { año } 2017 \text { (Capacitación) } \\
\text { 4. Becas Laborales año } 2017 \text { (Capacitación) }\end{array}$ & $\begin{array}{l}1997 \\
1999 \\
\end{array}$ & Eduardo Frei \\
\hline $\begin{array}{l}\text { 5. Aprendices año } 2017 \text { (Capacitación) } \\
\text { 6. Becas Fondo Cesantía Solidaria año } 2017 \\
\text { (Capacitación) } \\
\text { 7. Evaluación y Certificación de } \\
\text { Competencias Laborales año } 2017 \\
\text { (Capacitación) } \\
\text { 8. Subsidio al Empleo Joven año } 2017 \\
\text { (Subsidio) }\end{array}$ & $\begin{array}{l}2008 \\
2009 \\
2009\end{array}$ & Michelle Bachelet (primer periodo) \\
\hline $\begin{array}{l}\text { 10. Bono Empresa y Negocio } 2017 \\
\text { (Eprendimiento) }\end{array}$ & 2011 & \\
\hline $\begin{array}{l}\text { 11. Bono en el Trabajo de la Mujer año } 2017 \\
\text { (Subsidio) } \\
\text { 12. Capacitación en Oficios sectorial año } 2017\end{array}$ & $\begin{array}{l}2012 \\
2012\end{array}$ & Sebastián Piñera \\
\hline 13. + Capaz (Capacitación) & 2014 & Michelle Bachelet (segundo periodo) \\
\hline
\end{tabular}

Fuente: Elaboración propia a partir de información de SENCE

Como se observa, los programas Impulsa Personas (1976) y Transferencias al Sector Público (1988) comenzaron durante el periodo del gobierno militar. Al revisar las características de estos programas se puede inducir una gestión centrada en incentivar la capacitación entregando beneficios tributarios a las empresas y, por otra parte, favoreciendo a diversas instituciones públicas.

Posteriormente, los programas Capacitación en Oficios registro especial (1997) y Becas Laborales (1999), iniciados durante el gobierno de Eduardo Frei, dan cuenta de una gestión en capacitación cuyo objetivo principal fue promover la empleabilidad en grupos vulnerables (jóvenes y mujeres).

Así también se puede observar que seis de los doce programas vigentes a la fecha fueron creados durante el primer y segundo periodo de gobierno de la presidenta Michelle Bachelet: en su primer periodo comenzaron a funcionar Aprendices (2006), Becas Fondo Cesantía Solidaria (2007), Evaluación y Certificación de Competencias Laborales (2008), Subsidio al Empleo Joven año 2017 (2009), Fortalecimiento OMIL (2009), mientras que en el actual periodo se inició el programa +Capaz (2014). Este conjunto de programas responden a una mayor diversidad de objetivos y su foco de gestión no sólo apunta a capacitación, puesto que también al impulso de la empleabilidad en jóvenes, la disminución de la cesantía, la valoración de la 
experiencia laboral, el fortalecimiento de la institución pública a cargo de la inserción laboral, además del incentivo de la inserción laboral de grupos vulnerables (jóvenes, mujeres y discapacitados). De este modo, pareciera ser que en la concepción de estos programas ha existido una visión más integral respecto a la función social que puede cumplirse a través de la gestión en esta área.

Por último, durante el gobierno de Sebastián Piñera se aportó con la creación de tres programas denominados Bono Empresa y Negocio (2011), Bono en el Trabajo de la Mujer (2012) y Capacitación en Oficios sectorial (2012). Se podría decir que considerando los programas propuestos en este gobierno, se apostó a fortalecer el emprendimiento, la inserción femenina en el mercado de trabajo a través de subsidios y la capacitación dirigida sólo a sectores productivos de la pequeña y micro empresa.

\section{Inclusión/exclusión de la fuerza laboral a medida que envejece}

Finalmente, de acuerdo al grado de inclusión/exclusión de las personas a medida que envejecen, que es el eje de estudio, los programas se han clasificado en tres categorías: inclusivos (para todo rango etario), parcialmente inclusivos (con acceso hasta cierta edad o bajo ciertas condiciones) o no inclusivos (programa limitado a un rango o grupo específico solamente). El cuadro 5 resume esa clasificación.

\section{Cuadro 5 \\ Clasificación de los programas de acuerdo a su grado de inclusión de la fuerza laboral a medida que envejece y su focalización}

\begin{tabular}{|c|c|c|c|}
\hline & \multicolumn{3}{|c|}{ Programas } \\
\hline & No inclusivos & $\begin{array}{l}\text { Parcialmente } \\
\text { Inclusivos }\end{array}$ & Inclusivos \\
\hline $\begin{array}{l}\text { Exclusivamente para } \\
\text { población vulnerable }\end{array}$ & $\begin{array}{l}\text { Línea: Subsidios } \\
\text { - Bono de la mujer } \\
\text { - Subsidio al Empleo } \\
\text { Joven }\end{array}$ & $\begin{array}{l}\text { Línea: Capacitación } \\
\text { - Capacitación en } \\
\text { Oficios registro } \\
\text { especial } \\
\text { - Capacitación en } \\
\text { Oficios sectorial }\end{array}$ & $\begin{array}{l}\text { Línea: Capacitación } \\
\text { - Becas Fondo Cesantía } \\
\text { Solidaria } \\
\text { - Evaluacióny } \\
\text { Certificación de } \\
\text { Competencias } \\
\text { Laborales }\end{array}$ \\
\hline $\begin{array}{l}\text { Sin distinción en } \\
\text { población objetivo }\end{array}$ & & $\begin{array}{l}\text { Línea: Capacitación } \\
\text { - Transferencias al } \\
\text { Sector Público } \\
\text { - Capaz } \\
\text { - Aprendices } \\
\text { - Becas Laborales } \\
\text { Capacitación } 6\end{array}$ & $\begin{array}{l}\text { - ImpulsaPersonas } \\
\text { Línea: Empleo } \\
\text { - Fortalecimiento OMIL } \\
\text { Línea: } \\
\text { Emprendimiento } \\
\text { - Bono Empresay } \\
\text { Negocio }\end{array}$ \\
\hline
\end{tabular}

Fuente: Elaboración propia a partir de información de SENCE 
De acuerdo a lo que se observa en el cuadro precedente, los programas no inclusivos responden solamente a la línea de subsidios de intervención de SENCE. Los programas parcialmente inclusivos solamente son de la línea de capacitación y los inclusivos, por otra parte, responden a tres de las cuatro líneas: empleo, emprendimiento y capacitación.

Los programas no inclusivos están dirigidos únicamente a población vulnerable (mujeres en un rango de edad determinado y jóvenes). De los cinco programas parcialmente inclusivos, dos programas de estructura similar (Capacitación en Oficios registro especial y Capacitación en Oficios sectorial) están dirigidos a población vulnerable mientras que los tres restantes no son focalizados; dos de éstos (Aprendices y +Capaz) fueron creados durante gobiernos de Michelle Bachelet. Finalmente, de los cinco programas inclusivos, tres fueron creados en el gobierno de Michelle Bachelet (Becas Fondo Cesantía Solidaria, Evaluación y Certificación de Competencias Laborales y Fortalecimiento OMIL), uno en el gobierno de Sebastián Piñera (Bono Empresa y Negocio) y uno en el gobierno militar (Impulsa Personas).

\section{Discusión}

Los resultados de este estudio nos han permitido realizar una caracterización general de la oferta de programas que SENCE pone actualmente al servicio de la población de nuestro país en el ámbito del fomento del empleo y la capacitación. Las dimensiones abordadas fueron propuestas para poder ahondar en el eje principal de este estudio: la inclusión de las personas a medida que envejecen en las oportunidades de apoyo que brinda el estado en este ámbito, en un contexto social marcado por el envejecimiento acelerado de la población y con un panorama que augura dificultades crecientes desde el punto de vista económico a mediano plazo. Ese segmento de la población, con alta probabilidad, deberá permanecer en el mercado laboral hasta edades avanzadas, sea voluntariamente u obligados por la necesidad, requiriendo por tanto actualizar conocimientos, aprender nuevos oficios, sumar competencias.

\section{Capacitación: línea de intervención de mayor inversión en SENCE para el año 2016}

Desde el punto de vista descriptivo, un primer aspecto que llama la atención en el análisis es que de las cuatro categorías en que se clasifican los programas de SENCE, la que concentra la mayor parte de la oferta (nueve de los trece programas vigentes) es la de capacitación. Las otras categorías, que corresponden a apoyo al emprendimiento, empleo y subsidios suman solamente cuatro en total. De este modo, se puede señalar que la capacitación es la función más importante que orienta la gestión de esta institución y eso se refleja en el presupuesto destinado a esa línea de intervención. Con la información con la que se cuenta para el año 2016 y haciendo algunas interpolaciones a partir de datos disponibles, ${ }^{7}$ se estima que se 
destinaron para ese año un total aproximado a los 254.000 millones de pesos (US\$380.712.562 aproximadamente, al cambio de diciembre 2016) para efectos de esa categoría de programas. Esa cantidad es más del doble de lo que suman los otros cuatro programas, que fue un total aproximado de 119.100 millones de pesos (US\$178.515.221 aproximadamente, al cambio de diciembre 2016).

Para tener una mirada comparativa de lo destinado a capacitación, puede señalarse que corresponde a un $83,5 \%$ de lo que otra dependencia el Ministerio del Trabajo y Previsión Social -el IPS- destinó al pago de beneficios sociales correspondientes al Programa Chile Solidario, el Ingreso Ético Familiar y el Subsidio Único Familiar: 304.180 millones de pesos (US\$455.925.776 aproximadamente), tal como se señala en la Cuenta Pública del Ministerio del Trabajo y Previsión Social más reciente (Ministerio del Trabajo y Previsión Social, 2017).

\section{Vulnerabilidad social, mujeres y jóvenes: prioridades gubernamentales en empleo y capacitación}

Desde que se comenzaron a desarrollar estos programas hace 41 años los focos y destinatarios han ido variando. Llama la atención que, con posterioridad a la llegada de la democracia a Chile, de todos los programas vigentes cinco hayan sido creados en periodos de gobierno de Michelle Bachelet y que ninguno de los que existen en la oferta actual se haya iniciado en gobiernos de Patricio Aylwin o Ricardo Lagos.

Un elemento que podríamos denominar transversal a la lógica de definición de los programas es el de vulnerabilidad social: varios programas apuntan a sectores que están entre el 40 y $80 \%$ más vulnerable, lo cual es perfectamente coherente con lo que el SENCE tiene como misión, en la línea de contribuir a incrementar la productividad en la economía a través de medidas que impulsen la capacitación de los trabajadores de las empresas y también de las personas de bajos ingresos que no participan en el mercado laboral.

Las políticas de capacitación y empleo tienden a priorizar dos grupos en que focalizan sus acciones: mujeres y jóvenes. La empleabilidad de los jóvenes es una situación problemática en todo el orbe, lo cual también ha motivado análisis en América Latina. En un informe reciente se afirma que los jóvenes de nuestro continente enfrentan una elevada tasa de desempleo, que supera con creces la de los adultos (OIT, 2013) Las mujeres también se perciben como un grupo al cual hay que apoyar especialmente: la participación laboral femenina chilena, a pesar de haber aumentado significativamente desde los años noventa, es inferior a la media para América Latina (ILO, 2014) y la OCDE (OCDE, 2012), siendo las tasas generales de empleo en los últimos cinco años de un 66 -67\% para los hombres y sólo 44\% para las mujeres (INE, 2016).

Un tercer grupo de interés se ha ido incorporando recientemente a los programas de SENCE, lo cual resulta interesante y efectivamente cons- 
tituye una dimensión de inclusión, que aunque no está en el sentido de lo que se ha desarrollado en este estudio vale la pena ser destacado. Se trata de las personas discapacitadas, que han sido integradas en la población objetivo del programa Aprendices (2006) y +Capaz (2014). ${ }^{8}$

\section{Inclusión de la fuerza laboral que envejece en programas del SENCE: diagnóstico actual y propuestas}

Producto del análisis realizado en este estudio, se ha podido concluir que solamente cinco de los trece programas analizados son realmente inclusivos desde el punto de vista de la integración de la fuerza laboral a medida que envejece, sin distinciones respecto a grado de vulnerabilidad. Estos son:

Línea: Capacitación

- Becas Fondo Cesantía Solidaria

- Evaluación y Certificación de Competencias Laborales

- Impulsa Personas

Línea: Empleo

- Fortalecimiento OMIL

Línea: Emprendimiento

- Bono Empresa y Negocio

Hay varios programas que se han considerado potencialmente inclusivos, que aunque no están abiertos a todo tipo de destinatario con relación a la edad en determinadas circunstancias sí abren opciones para personas mayores. Uno de los casos que vale la pena destacar es de la las Becas Laborales, que comprende una línea específica de inclusión laboral para personas mayores de 64 años. Otro programa que ha ampliado el acceso de manera inclusiva es Aprendices, en este caso dirigido a personas que conjugan dos aspectos clave en la inclusión: discapacidad y envejecimiento. Este último programa fue analizado -en versiones análogas - en un estudio que revisó su implementación en varios países: mientras en versiones que existen en Alemania, Estados Unidos, Canadá y otros países avanzados en materia laboral se admiten personas de toda edad, en Perú, Brasil y Chile la tendencia es la focalización en población joven, sector que se califica como crítico en materia de empleabilidad (Fazio, Fernández-Coto, \& Ripani, 2016).

Este estudio constituye una aproximación exploratoria a un tema clave desde el punto de vista de la integración de la fuerza laboral que está envejeciendo en Chile. Las oportunidades de capacitación para este grupo, aunque existen, son insuficientes. Tal como plantean diversos autores, para las personas mayores que decidan mantenerse en el mercado laboral o se vean obligados por razones económicas, la exclusión laboral y la discriminación se pueden evitar si están suficientemente capacitados y actualizados en conocimientos (Eichhorst et al., 2013; Cepeda, 2010; Ghosheh, 2008) 
Hay diversos instrumentos que pueden mejorarse, ampliarse en su cobertura. Además, es posible diseñar nuevos programas que respondan a los requerimientos de este sector. Esta tarea constituye sin duda un gran desafío para la agenda laboral de quienes encabecen el próximo gobierno.

\section{Agradecimientos}

Este artículo forma parte del trabajo realizado en la tesis para optar al grado de Doctor en Psicología en la Pontificia Universidad Católica de Valparaíso de la autora principal. 


\section{Notas}

${ }^{1}$ En su tercera versión se sumó Flacso Chile.

${ }^{2}$ De acuerdo a los autores del estudio, los programas de aprendices tienen cuatro características distintivas: i) el aprendiz tiene un trabajo con capacitación estructurada; ii) el plan de formación combina capacitación en el lugar de trabajo a cargo de un maestro de la profesión y capacitación en aula; iii) existe un contrato de empleo escrito entre empleador y aprendiz, donde se establecen derechos y responsabilidades de ambas partes; y iv) el aprendiz debe aprobar una evaluación para recibir una certificación. La implementación de este tipo de modelo varía mucho entre los distintos países.

${ }^{3}$ Uno de los elementos centrales en el concepto de focalización es la vulnerabilidad social. La información con la que se construyen los tramos de calificación socioeconómica en el país proviene de varias fuentes administrativas del Estado y se consideraran además los antecedentes que aporten las personas. Se distinguen tramos que van desde el $40 \%$ calificados de menores ingresos -o mayor vulnerabilidad- hasta el $91 \%$ al $100 \%$ calificados de mayores ingresos -o menor vulnerabilidad-. Para más detalle, puede revisarse el Informe de Desarrollo Social en el sitio http:// www.ministeriodesarrollosocial.gob.cl/pdf/upload/Informe_de_ Desarrollo_Social_2016.pdf

4 Un elemento diferenciador de este programa es que explicita una línea de acción orientada a beneficiar específicamente a personas mayores de 64 años en su inclusión laboral.

5 Tiene la particularidad de que sus beneficiarios no son directamente personas, sino más bien las entidades municipales encargadas de gestionar la intermediación laboral de todas las personas que lo soliciten, sin diferenciación de sexo, edad o grupo social.

6 Si bien este programa tiene como público objetivo personas de toda edad, dado que está dirigido solamente al $80 \%$ de la población más vulnerable se le considera parciamente inclusivo

${ }^{7}$ Como uno de los programas de capacitación, Impulsa Personas, no cuenta con información sobre presupuesto asignado para el 2016, se usó el valor del año 2015 para estimar el total de la categoría

8 El 8 de junio del presente año se promulgó la Ley de Inclusión Laboral, que entre otras normativas, elimina la posibilidad de pagar menos que el sueldo mínimo a trabajadores con discapacidad mental; instaura de manera explícita la prohibición de todo acto de discriminación que se traduzca en exclusiones basadas en motivos de discapacidad; fija en 26 años la edad límite para suscribir contrato de aprendizaje con personas en situación de discapacidad; e incorpora formalmente la obligación de los Ministerios del Trabajo y Previsión Social y de Desarrollo Social de evaluar el cumplimiento del Sistema de Inclusión Laboral cada 4 años. 


\section{Bibliografía}

Atkinson, P., \& Coffey, A. (2006). Analysing documentary realities. En D. Silverman (Ed.). Interpreting Qualitative Data. (pp. 45-62) Londres, U.K: SAGE Publications.

Bravo, J. (2016). Adultos mayores: segmento clave en la evolución reciente del mercado laboral. Informe laboral, CLAPES UC. Recuperado de http://www.clapesuc.cl/assets/uploads/2016/03/22-02-16-situacionlaboral-oct-dic-2015.pdf

CEPAL (2012). Eslabones de la Desigualdad. Heterogeneidad estructural, empleo y protección social. Recuperado de: http://www.eclac.cl/publicaciones/xml/2/47382/Eslabones_de_la_desigualdad.pdf

CEPAL (2017). Agenda 2030 y los Objetivos de Desarrollo Sostenible: una oportunidad para América Latina y el Caribe. Santiago: Naciones Unidas. Recuperado de http://repositorio.cepal.org/handle/11362/ 40155

Comisión Revisora del Sistema de Capacitación e Intermediación Laboral (2011). Informe Final. Recuperado de http://www.cl.undp.org/ content/dam/chile/docs/pobreza/undp_cl_pobreza _InformeFinal_211011_doc2.pdf

Ministerio de Desarrollo Social, Servicio Nacional del Adulto Mayor. (2012). Política integral de envejecimiento positivo para chile 2012-2025. ISBN 978-956-8846-02-2

Ministerio del Trabajo y Previsión Social, Servicio Nacional de Capacitación y Empleo. (2017). + Capaz “año 2017”. Recuperado de http:/ /www.sence.cl/601/articles-7412_archivo_01.pdf

Ministerio del Trabajo y Previsión Social, Servicio Nacional de Capacitación y Empleo. (2017). “Aprendices año 2017”. Recuperado de http:/ /www.sence.cl/601/articles-7412_archivo_02.pdf

Ministerio del Trabajo y Previsión Social, Servicio Nacional de Capacitación y Empleo. (2017). “Becas Fondo Cesantía Solidario año 2017”. Recuperado de http://www.sence.cl/601/articles-7412_archivo_03.pdf

Ministerio del Trabajo y Previsión Social, Servicio Nacional de Capacitación y Empleo. (2017). “Becas Laborales año 2017”. Recuperado de http://www.sence.cl/601/articles-7412_archivo_04.pdf

Ministerio del Trabajo y Previsión Social, Servicio Nacional de Capacitación y Empleo. (2017). “Bono al Trabajo de la Mujer año 2017”. Recuperado de http://www.sence.cl/601/articles-7412_archivo_05.pdf

Ministerio del Trabajo y Previsión Social, Servicio Nacional de Capacita- 
ción y Empleo. (2017). “Bono Empresa y Negocio 2017”. Recuperado de http://www.sence.cl/601/articles-7412_archivo_06.pdf

Ministerio del Trabajo y Previsión Social, Servicio Nacional de Capacitación y Empleo. (2017). "Capacitación en Oficios registro especial año 2017”. Recuperado de http://www.sence.cl/601/articles7412_archivo_07.pdf

Ministerio del Trabajo y Previsión Social, Servicio Nacional de Capacitación y Empleo. (2017). “Evaluación y Certificación de Competencias Laborales año 2017”. Recuperado de http://www.sence.cl/601/ articles-7412_archivo_09.pdf

Ministerio del Trabajo y Previsión Social, Servicio Nacional de Capacitación y Empleo. (2017). “Fortalecimiento OMIL año 2017”. Recuperado de http://www.sence.cl/601/articles-7412_archivo_10.pdf

Ministerio del Trabajo y Previsión Social, Servicio Nacional de Capacitación y Empleo. (2017). “Subsidio al Empleo Joven año 2017”. Recuperado de http://www.sence.cl/601/articles-7412_archivo_11.pdf

Ministerio del Trabajo y Previsión Social, Servicio Nacional de Capacitación y Empleo. (2017). “Transferencias al Sector Público 2017”. Recuperado de http://www.sence.cl/601/articles-7412_ archivo_12.pdf

Ministerio del Trabajo y Previsión Social, Servicio Nacional de Capacitación y Empleo. (2017). “Impulsa Personas año 2017”. Recuperado de http://www.sence.cl/601/articles-7412_archivo_13.pdf

Ministerio del Trabajo y Previsión Social, Servicio Nacional de Capacitación y Empleo. (2017). “Capacitación en Oficios sectorial año 2017””. Recuperado de http://www.sence.cl/601/articles7412_archivo_08.pdf

Ministerio del Trabajo y Previsión Social, Servicio Nacional de Capacitación y Empleo. (s. f). Acerca del Sence. Recuperado de http:// www.sence.cl/portal/Acerca-del-Sence/

Ministerio del Trabajo y Previsión Social. (2017). Cuenta Pública Participativa. Informe de Gestión 2016 y Desafíos 2017. Recuperado de http://www.subtrab.trabajo.gob.cl/wp-content/uploads/2017/05/ CUENTA-PUBLICA-1.pdf

Didier, N., \& Cox, J. (2012). International briefing 25: Training and development in Chile. International Journal of Training and Development, 16(2), 145-154. doi: 10.1111/j.14682419.2012.00399.x 
Didier, N., Pérez, C., \& Valdenegro, D. (2013). Capacitación y capital humano: análisis de las últimas dos décadas. Revista de Psicología, 22(2), 87-99. Doi: 10.5354/0719-0581.2013.30856

Fazio, M., Fernández-Coto, R., \& Ripani, L. (2016). Aprendices para el siglo XXI ¿Un modelo para América Latina y el Caribe?. Banco Interamericano del Desarrollo. Recuperado de http:// www.oitcinterfor.org/node/6959

Ganga, F., Piñones, M., González, D. \& Rebagliati, F. (2016). Rol del Estado frente al envejecimiento de la población: el caso de Chile. Convergencia, 23(71), 175-200. Recuperado de http:// www.scielo.org.mx/scielo.php?script=sci_arttext\&pid=S1405$14352016000200175 \& \operatorname{lng}=$ es\&tlng=es.

ILO (2014). Global employment trends 2014: Risk of a jobless recovery? Geneva: ILO. ISBN 978-92-2-127486-5

Instituto Nacional de Estadísticas (INE) - Chile. (2016) Nueva encuesta nacional de empleo (NENE) [Internet]. Recuperado de www.ine.cl

Marcel, M., \& Naudon, A. (2016). Transiciones laborales y la tasa de desempleo en Chile. Documento de Trabajo $N^{\circ} 787$, Banco Central de Chile. Recuperado de http://si2.bcentral.cl/public/pdf/documentostrabajo/pdf/dtbc787.pdf

Mardones, C., \& Sepúlveda, L. (2017). Evaluación ex-post de franquicia tributaria SENCE para capacitación laboral y su efecto sobre empresas chilenas. Estudios Gerenciales, 33, 163-176. Doi: 10.1016/ j.estger.2017.03.001

Mesa-Lago, C. (2015). Reformas estructurales y Re-reformas de pensiones, y sus efectos en el bienestar social: El caso de Chile. Política y Sociedad, 52(3), 719-739. Doi: http://dx.doi.org/10.5209/ rev_POSO.2015.v52.n3.45378

OECD (2012) Closing the Gender Gap: Act Now. Paris: OECD Publishing. ISBN 978-92-64-17937-0

OECD. (2015). Pensions at a Glance 2015. Recuperado de http:// www.oecd.org/publications/oecd-pensions-at-a-glance-19991363.htm

OECD. (2016). Net pension replacement rates (indicator). Doi: 10.1787/ 4b03f028-en.

OIT (2013). Tendencias mundiales de empleo juvenil 2013. Una generación en peligro. Ginebra: OIT. ISBN 978-92-2-327484-9 
OIT. (2016). Panorama Laboral América Latina y el Caribe. Lima: OIT / Oficina Regional para América Latina y el Caribe.

Pavez, J., Aguilar, C., Peña, M., de los Ángeles Bilbao, M., Oriol, X., Ortúzar, H., \& Torres, J. (2016). Sujeto/a trabajador/a en la política pública de intervención psicosocial de infancia en chile: un análisis crítico del discurso. Revista Estudios Cotidianos, 4(1), 23-45.

Programa Adulto Mayor UC y Centro UC Estudios de Vejez y Envejecimiento. (2017). Chile y sus mayores. 10 años de la Encuesta Calidad de Vida en la Vejez UC - Caja Los Andes. Resultados IV Encuesta Calidad de Vida en la Vejez. Santiago: Fyrma Gráfica. No 978-956-14-2059-5

Rivera-Aguilera, G. (2016). Gobernar a la juventud vulnerable: un análisis discursivo de las políticas de empleo juvenil en Chile. Pueblos y fronteras, 11(21), 192-213.

Sisto, V., \& Fardella, C. (2011). Nuevas políticas públicas, epocalismo e identidad: El caso de las políticas orientadas a los docentes en Chile. Revista de Estudios Universitarios, 37(1), 123-141.

Sojo, A. (2014). El sistema contributivo de pensiones como locus de rivalidad y de un nuevo pacto social en Chile. Santiago de Chile: Naciones Unidas. ISSN 1564-4162

Thumala, D., Arnold, M., Massad, C., \& Herrera, F. (2015). Inclusión y exclusión social de las personas mayores en Chile. Santiago: SENAMA-FACSO U. de Chile Ediciones.

Torres, M. (2016). Capacitación Laboral, Trabajo de Calidad y Exclusión Social. (Memoria para optar al título de sociólogo). Universidad de Chile, Santiago, Chile.

Vives, A., Molina, A., Gray, N., \& González, F. (2017). Envejecimiento y trabajo en Chile: Propuesta para el monitoreo de la salud laboral. En Irarrázaval, I.; Piña, E. \& Letelier, M. (Eds). Propuestas para Chile. Concurso Políticas Públicas 2016. Santiago: Salesianos Impresores, pp. 17- 49 .

Weller, J. (2001). Procesos de exclusión e inclusión laboral: la expansión del empleo en el sector terciario. Santiago: Naciones Unidas. ISBN: 92-1- 321949-0

Weller, J. (2014). Aspectos de la evolución reciente de los mercados laborales de América Latina y el Caribe. Revista Cepal, 114. Recuperado de http://repositorio.cepal.org/bitstream/handle/11362/37434/ RVE114Weller_es.pdf 
Polis, Revista Latinoamericana, $N^{\circ} 48,2017$

WHO (2002). Active ageing: a policy framework. Geneva: World Health Organization.

Recibido: 15.09.17

Aceptado: 03.11 .17 